\title{
ANÁLISE DA TRAJETÓRIA DE UM MÍSSIL BALÍSTICO
}

\author{
LUÍS OTÁVIO MARCHI, CARLOS RENATO HUAURA SOLORZANO
}

\author{
Centro de Engenharia, Modelagem e Ciências Sociais Aplicadas, Universidade Federal do ABC \\ Avenida dos Estados, 5001 - Bangu - CEP 09.210-170 - Santo André - SP - Brasil \\ E-mails: marchi.luis@yahoo.com.br,carlos.solorzano@ufabc.edu.br
}

\begin{abstract}
Resumo_- Programa Espacial Brasileiro começou a ser projetado em 1961, quatro anos após o lançamento do primeiro satélite o Sputnik 1. Nesses cinqüenta anos foram criados centros de pesquisas para o desenvolvimento de projetos espaciais que incluem a criação de foguetes, satélites e bases de lançamento. Hoje, várias instituições brasileiras, governamentais e privadas, desenvolvem pesquisas na área de tecnologia espacial para a produção de foguetes e mísseis. Do ponto de vista de defesa nacional existem diversas empresas voltadas a fabricação de mísseis, tais como Avibrás e Mectron (Organização Odebrecht). Desta forma a tecnologia de mísseis tem evoluído aceleradamente desde os seus inícios dado pelo primeiro foguete balístico V2. Nos dias de hoje diversos estudos são realizados voltados a trajetória, estrutura, controle, eletrônica, propulsão e guiagem de mísseis. O presente trabalho pretende abordar a análise da trajetória de um míssil balístico considerando efeitos gravitacionais e de arrasto atmosférico.
\end{abstract}

Palavras-chave—astrodinamica, trajetória, missil balistico

\section{Introdução}

Do ponto de vista da defesa nacional existem algumas empresas voltadas a produção de produtos bélicos. A Avibras Indústria Aeroespacial e a Mectron (Organização Odebrecht) atuam no desenvolvimento e fabricação de produtos de alta tecnologia e valor agregado, tanto para uso civil como militar. Trata-se de sistemas complexos como armamentos inteligentes, sistemas aviônicos, radares, equipamentos para satélites entre outros componentes que exigem capacitações técnicas nas mais diversas áreas da engenharia. É nítido o interesse do governo brasileiro em manter em pleno funcionamento as empresas especializadas em produtos bélicos, pois os mercados internacionais globalizados podem transformar-se em mercados consumidores fortalecendo as exportações.

Além disso, este tipo de tecnologia possibilitaria um alto poder de dissuasão às ações internacionais comprometedoras a integridade nacional que, por ventura, estivessem a fim de usufruir dos recursos naturais recentemente descobertos e atualmente cobiçados devido ao alto valor econômico agregado.

\section{Modelos Matemáticos}

A trajetória de um míssil balístico é composta basicamente de três etapas na qual a primeira delas é o voo propulsionado que se refere desde o momento de lançamento até o corte de empuxo no ponto final de queima, a segunda etapa é composta pelo período de voo livre que constitui a maior parte da trajetória e, a reentrada, que começa em um ponto indefinido da atmosfera na qual a força de arrasto deve ser levada em consideração, até o ponto de impacto. Sabe-se que a energia é continuamente adicionada ao míssil durante a fase de voo propulsionado, o que impossibilita o uso da mecânica de 2 corpos para determinar o caminho entre o lançamento até o ponto final de queima. A trajetória do míssil nesta parte crítica de voo é determinada pelo sistema de guiagem e nave- gação que a princípio não pertence ao escopo desta pesquisa. Em contrapartida, no voo livre a trajetória é parte de uma órbita cônica - quase sempre uma elipse - que podemos analisar usando os princípios da mecânica envolvendo 2 corpos. $\mathrm{Na}$ reentrada existe a dissipação de energia por atrito com a atmosfera.

As hipóteses iniciais a serem assumidas são basicamente: planeta Terra como irrotacional (ausência de Rotação), altitudes de ponto final de queima e reentrada iguais.

A formulação matemática inicia-se com a definição de um parâmetro adimensional, $Q$, expresso em função do parâmetro gravitacional, $\mu$, como:

$$
Q \equiv \frac{v^{2} r}{G M_{\text {terra }}}=\frac{v^{2} r}{\mu}
$$

- $G=6,67384 \times 10^{-11} \mathrm{~m}^{3} /\left(\mathrm{kg} \mathrm{s}^{2}\right)$ e $M_{\text {terra }}=5,972 \times 10^{24} \mathrm{~kg}$.

Na Figura 1 é possível identificar a geometria da trajetória e as variáveis de interesse para realização dos cálculos (Bate et al. 1971).

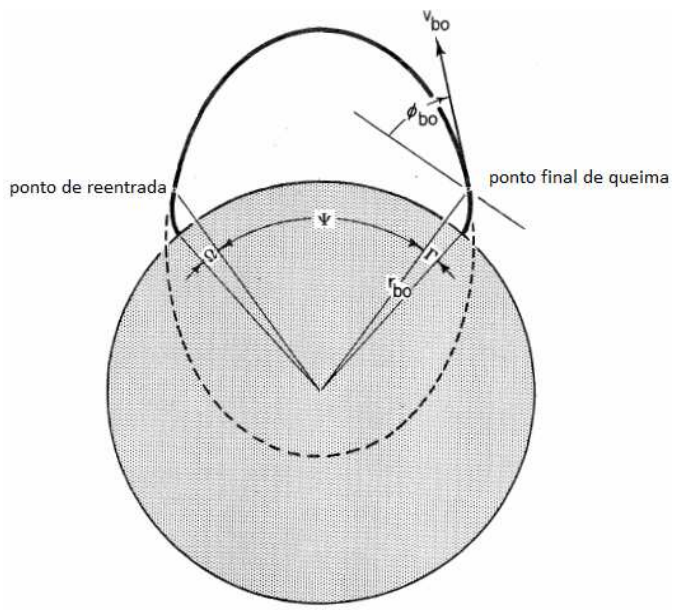

Figura 1. Geometria da trajetória de um míssil balístico. 
Os parâmetros são:

$\Gamma$ - ângulo de alcance de voo propulsionado;

$\Psi$ - ângulo do alcance de voo livre;

$\Omega$ - ângulo do alcance de reentrada;

$\Lambda$ - ângulo de alcance total $(\Gamma+\Psi+\Omega)$;

$\mathrm{V}_{\mathrm{bo}}$ - velocidade no ponto final de queima;

$\mathrm{r}_{\mathrm{bo}}$ - distância entre o centro da Terra ao ponto final de queima;

$\Phi_{\text {bo }}$ ângulo de caminho de voo no ponto final de queima;

Visto que a trajetória de voo livre de um míssil é uma seção cônica, a equação geral das cônicas pode ser aplicada no ponto final de queima.

$$
r_{b o}=\frac{p}{1+e \cos \left(v_{b o}\right)}
$$

- $e$ : excentricidade da trajetória;

$\bullet p$ : semilatus rectum;

- $v_{b o}$ : anomalia verdadeira no ponto final de queima;

Ao isolar cos $\left(v_{\mathrm{bo}}\right)$, obtém-se:

$$
\cos \left(v_{b o}\right)=\frac{p-r_{b o}}{e r_{b o}}
$$

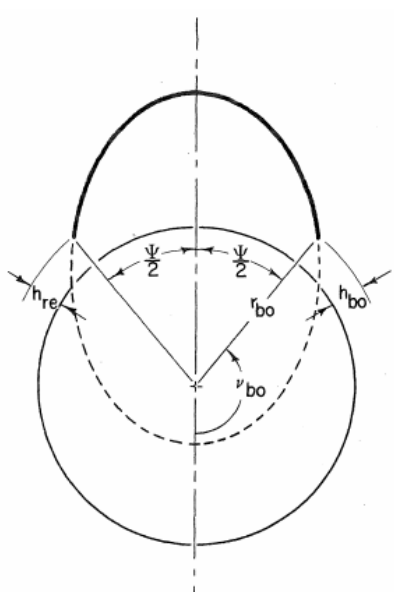

Figura 2. Trajetória Simétrica.

- $\quad \mathrm{h}_{\mathrm{bo}}$ : altitude do burnout;

- $\mathrm{h}_{\mathrm{re}}$ : altitude de re-entrada;

Ao assumir que a trajetória de voo livre deve ser simétrica $\left(\mathrm{h}_{\mathrm{bo}}=\mathrm{h}_{\mathrm{re}}\right)$, metade do ângulo de alcance de voo livre, $\psi$, encontra-se em cada lado do semi-eixo maior.

$$
\cos \left(\frac{\psi}{2}\right)=-\cos \left(v_{b o}\right)
$$

Portanto, a Eq. (3) pode ser reescrita como:

$$
\cos \left(\frac{\psi}{2}\right)=\frac{r_{b o}-p}{e r_{b o}}
$$

Esta última expressão para o ângulo de alcance de voo livre esta em função de $p, e, r_{b o}$. Porém, $p=h^{2} / \mu \mathrm{e}$ $h=r v \cos \Phi$ ( $h$ : momento angular). Ao utilizar a definição de $Q$ (Eq. 1) chega-se:

$$
p=\frac{r^{2} v^{2} \cos ^{2} \phi}{\mu}=r Q \cos ^{2} \phi
$$

Agora, uma vez que, $p=a\left(1-e^{2}\right)$, então:

$$
e^{2}=1-\frac{p}{a}
$$

Ao substituir $p=r Q \cos ^{2} \Phi$ e $a=r /(2-Q)$, tem-se:

$$
e^{2}=1+Q(Q-2) \cos ^{2}(\phi)
$$

Se substituirmos as Eq. (6) e (8) na Eq. (5) teremos uma nova forma de expressar a equação de alcance de voo livre para as condições expressas no ponto final de queima.

$$
\cos \left(\frac{\psi}{2}\right)=\frac{1-Q_{b o} \cos ^{2} \phi_{b o}}{\sqrt{\left(1+Q_{b o}\left(Q_{b o}-2\right) \cos ^{2} Q_{b o}\right)}}
$$

Na Figura 3 nós temos o desenho do horizonte local bem como a tangente e a normal no ponto de burnout. A linha do ponto de burnout ao foco secundário, F', é chamada r' bo.

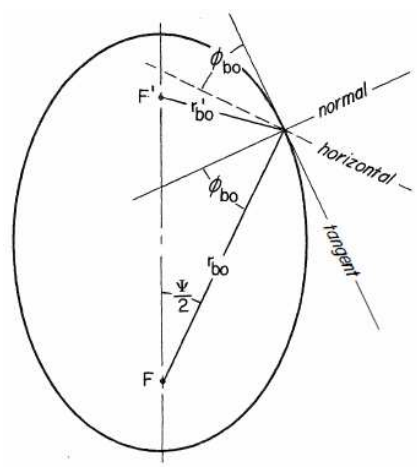

Figura 3. Geometria da elipse. 
O ângulo entre o horizonte local e a tangente (direção de $v_{b o}$ ) é o ângulo de caminho de voo, $\Phi_{b o}$. Uma vez que $r_{b o}$ é perpendicular ao horizonte local e a normal é perpendicular a tangente, o ângulo entre $r_{b o}$ e a normal também será $\Phi_{b o}$. Depois de algum algebrismo é possível provar que o ângulo entre $r_{b o}$ e $r_{b o}^{\prime}$ é $2 \Phi_{b o}$. Agora, basta se concentrar no triângulo formado por $F, F$ ' e o ponto de burnout. É conhecido dois ângulos deste triângulo e o terceiro pode ser determinado pelo simples fato de que a soma dos ângulos internos de um triângulo é $180^{\circ}$.

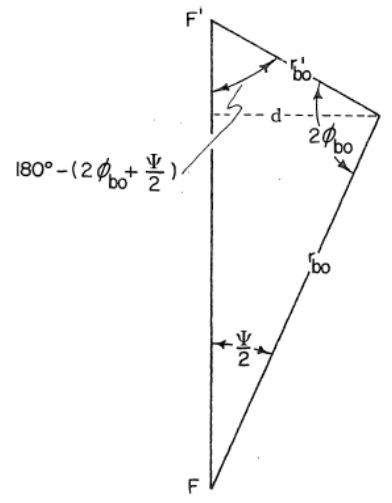

Figura 4. Triângulo formado pelos focos e o ponto final de queima.

Ao dividir este triângulo em dois triângulos retângulos por uma linha tracejada, $d$, como mostrado na Figura 4, pode-se expressar $d$, como:

$$
d=r_{b o} \operatorname{sen}\left(\frac{\psi}{2}\right)
$$

ou ainda:

$$
d=r_{b o}^{\prime} \operatorname{sen}\left[180^{\circ}-\left(2 \phi_{b o}+\frac{\psi}{2}\right)\right]
$$

Ao combinar as Eqs. (10) e (11) e lembrando que $\operatorname{sen}\left(180^{\circ}-x\right)=\operatorname{sen}(x)$ :

$$
\operatorname{sen}\left(2 \phi_{b o}+\frac{\psi}{2}\right)=\frac{r_{b o}}{r_{b o}^{\prime}} \operatorname{sen}\left(\frac{\psi}{2}\right)
$$

Contudo, $r_{b o}=a\left(2-Q_{b o}\right)$ e $r_{b o}+r_{b o}^{\prime}=2 a$, o que possibilita chegar na equação de ângulo de caminho de voo.

$$
\operatorname{sen}\left(2 \phi_{b o}+\frac{\psi}{2}\right)=\frac{2-Q_{b o}}{Q_{b o}} \operatorname{sen}\left(\frac{\psi}{2}\right)
$$

Ao derivar parcialmente a Eq. (13) em relação à $\Phi_{b o}$ e após isolar $\partial \psi / \partial \Phi_{b o}$, chega-se em:

$$
\frac{\partial \psi}{\partial \phi_{b o}}=\frac{-4 \cos \left(2 \phi_{b o}+\frac{\psi}{2}\right)}{\cos \left(2 \phi_{b o}+\frac{\psi}{2}\right)-\left(\frac{2-Q_{b o}}{Q_{b o}}\right) \cos \left(\frac{\psi}{2}\right)}
$$

Agora, basta igualar a Eq. (14) à zero. Deste modo determina-se $\Phi_{b o}$ como sendo:

$$
\phi_{b o}=\frac{1}{4}(\pi-\psi)
$$

ou seja,

$$
2 \phi_{b o}+\frac{\psi}{2}=\frac{\pi}{2}
$$

Ao substituir a equação (16) na Eq. (13) e isolando $Q_{b o}$ :

$$
Q_{b o}=\frac{2 \operatorname{sen}\left(\frac{\psi}{2}\right)}{1+\operatorname{sen}\left(\frac{\psi}{2}\right)}
$$

Esta equação é útil para determinar o menor valor de $Q_{b o}$ que irá atingir um determinado ângulo $\psi$.

Por simetria, visualiza-se através da Figura 5, que o tempo de voo do ponto final de queima para o ponto de reentrada é duas vezes o tempo de voo do ponto final de queima (ponto1) para o apogeu (ponto2).

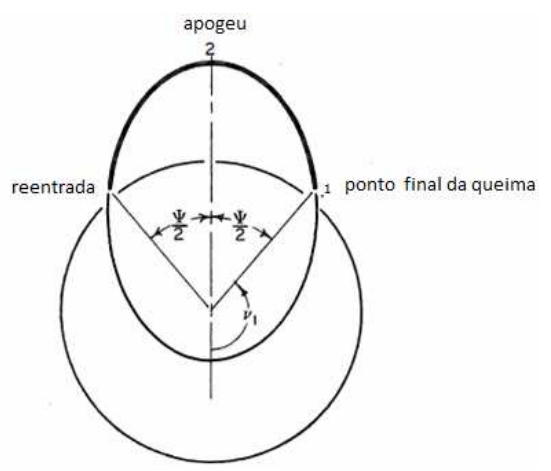

Figura 5. Tempo de voo livre.

A anomalia excêntrica no ponto 2 é $\pi$ radianos ou $180^{\circ}$. Em relação ao ponto 1 a anomalia pode ser computada notando que $v_{1}=180^{\circ}-(\psi / 2)$.

$$
\cos \left(E_{1}\right)=\frac{e-\cos \left(\frac{\psi}{2}\right)}{1-e \cos \left(\frac{\psi}{2}\right)}
$$

Com isso, é possível expressar o tempo de voo livre em função do semi-eixo maior $(a)$, parâmetro 
gravitacional $(\mu)$ e anomalia excêntrica no ponto 1 $\left(\mathrm{E}_{1}\right)$.

$$
t_{f f}=2 \sqrt{\frac{a^{3}}{\mu}}\left(\pi-E_{1}+e \operatorname{sen} E_{1}\right)
$$

Existem dois modelos físicos (Zarchan, 1997) que levam em consideração à influência gravitacional e que podem ser utilizados no estudo de trajetórias balísticas. $\mathrm{O}$ mais simples deles é conhecido como Modelo Terra Plana o qual a aceleração da gravidade é considerada constante independentemente da altitude. Assim, sua validade se restringe apenas a pequenas altitudes uma vez que a influência gravitacional sobre o corpo diminui com a altitude. Já o segundo modelo baseia-se na equação do problema de dois corpos o qual leva em consideração a variação da gravidade com a altitude.

Além do gradiente gravitacional o efeito do arrasto atmosférico também deve ser levado em consideração. Apesar de mísseis balísticos de longo alcance ficarem a maior parte do tempo na condição de voo livre (atmosfera rarefeita), na fase de voo propulsionado (ponto de lançamento ao ponto final de queima) e na fase de reentrada (altitude de reentrada até o impacto com o alvo) há a presença da resistência atmosférica que leva a diminuir a capacidade de alcance. À vista disso, é necessário inserirmos nos cálculos a força de arrasto sendo dada como:

$$
F_{\text {arrasto }}=Q S_{\text {ref }} C_{D 0}
$$

- $\quad Q$ : pressão dinâmica;

- $\quad S_{\text {ref: área de referência; }}$

- $\quad C_{d 0}$ : coeficiente de arrasto quando a sustentação aerodinâmica é nula;

A pressão dinâmica é função da densidade do ar $\rho$ e da velocidade do míssil $V$, portanto:

$$
Q=\frac{\rho V^{2}}{2}
$$

A área de referência relaciona-se à seção transversal do corpo do míssil e, por esse motivo, está associada com suas características físicas. Já o coeficiente de arrasto é função da velocidade e forma aerodinâmica do míssil.

Como a densidade do ar diminui com a altitude, a influência do arrasto é muito maior a baixas altitudes.

O Padrão Atmosférico Norte Americano contém uma série de modelos que definem valores para a temperatura atmosférica, pressão, densidade e outras propriedades que variam ao longo de uma ampla faixa de altitude. $\mathrm{O}$ primeiro modelo foi publicado em 1958 e foi atualizado em 1962, 1966 e 1976. Em primeira análise a aproximação exponencial de 1962 oferece boa precisão para os cálculos da densidade.

$$
\begin{gathered}
\rho=0.002378 e^{(- \text {alt } / 9144)} \quad \text { se } \text { alt }<9144 \mathrm{~m} \\
\rho=0.0034 e^{(- \text {alt } / 6706)} \text { se } \text { alt } \geq 9144 \mathrm{~m}
\end{gathered}
$$

- $\quad$ alt: altitude a qual se encontra o míssil;

Portanto, é importante observar que a magnitude da desaceleração devido ao arrasto é influenciada por $\mathrm{C}_{\mathrm{D} 0}$ e a altitude. Desta maneira, é conveniente combinar o peso total $(W), \mathrm{S}_{\mathrm{ref}}$ e $\mathrm{C}_{\mathrm{D} 0}$ em uma única expressão denominada coeficiente balístico $(\beta)$.

$$
\beta=\frac{W}{C_{D 0} S_{r e f}}
$$

As equações diferenciais agindo no míssil são:

$$
\begin{gathered}
\dot{V}_{x}=-a_{\text {arrasto }} \cos (\phi) \\
\dot{V}_{y}=-g-a_{\text {arrasto }} \operatorname{sen}(\phi) \\
\dot{R}_{x}=V_{x} \\
\dot{R}_{y}=V_{y}
\end{gathered}
$$

- $\quad V$ : velocidade do míssil;

- $\quad R$ : alcance do míssil;

As condições iniciais para velocidade e posição são dadas por:

$$
\begin{gathered}
V_{x}(0)=V \cos (\phi) \\
V_{y}(0)=V \operatorname{sen}(\phi) \\
R_{x}(0)=d r \\
R_{y}(0)=\text { alt }
\end{gathered}
$$

Por fim, o módulo da aceleração devido ao arrasto atmosférico pode ser escrito como sendo:

$$
a_{\text {arrasto }}=\frac{Q g}{\beta}=\frac{1}{2} \rho V^{2} C_{D 0} \frac{S}{m}
$$

\section{Resultados}

É possível expressar graficamente cinco parâmetros de interesse $\left(Q, e, \psi, \Phi\right.$ e $\left.\mathrm{t}_{\mathrm{ff}} / \mathrm{P}_{\mathrm{cs}}\right)$ onde $\mathrm{P}_{\mathrm{cs}}$ é o período circular calculado na altitude do burnout como:

$$
P_{C S}=2 \pi \sqrt{\frac{r_{b o}^{3}}{\mu}}
$$


A lógica de programação exige a obtenção de equações na qual todos os parâmetros, cujas curvas serão geradas, devem ser expressos em função de outro parâmetro constante e de $\psi$. Em poucas palavras, se $\Phi$ é constante, Q pode ser escrito em função do próprio $\Phi$ e da variável de interesse $\psi$. O mesmo raciocínio foi utilizado em relação aos demais parâmetros conforme o esquema abaixo.

$1^{\circ}$ caso:

$$
\phi=\text { const. } \rightarrow Q(\phi, \psi)
$$

$$
Q=\frac{2 \operatorname{sen}\left(\frac{\psi}{2}\right)}{\operatorname{sen}\left(2 \phi+\frac{\psi}{2}\right)+\operatorname{sen}\left(\frac{\psi}{2}\right)}
$$

$2^{\circ}$ caso

$$
\begin{gathered}
Q=\text { const. } \rightarrow \phi(Q, \psi) \\
\phi=\frac{\operatorname{asen}\left(\frac{2-Q}{Q} \operatorname{sen} \frac{\psi}{2}\right)}{2}-\frac{\psi}{4}
\end{gathered}
$$

$3^{\circ}$ caso

$$
\begin{gathered}
e=\text { const. } \rightarrow Q(e, \psi) \\
Q=\frac{-e^{2}-1+2 e \cos \left(\frac{\psi}{2}\right)}{e \cos \left(\frac{\psi}{2}\right)-1}
\end{gathered}
$$

A Figura 5 contém o tempo de voo versus o ângulo de alcance para diversos valores de excentricidade $(e)$, parâmetro adimensional $(Q)$ e ângulo caminho de voo $(\Phi)$.

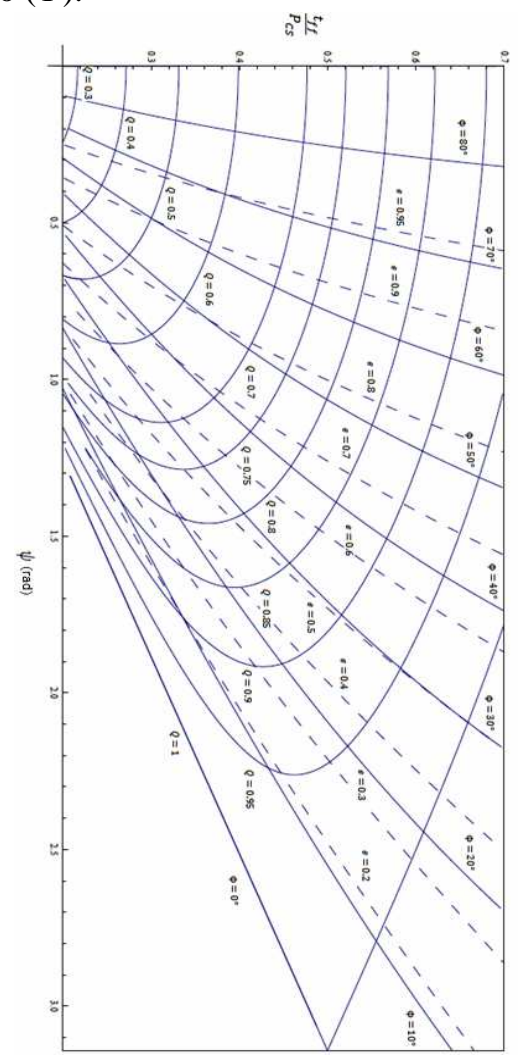

Figura 5. Tempo de voo vs ângulo de alcance.
Ao inserir a componente de arrasto nas equações do Modelo Terra Plana (ver Eq. 25 a 28) o alcance e altitude sofrem brusca variação. A Figura 8 contém os resultados para um lançamento efetuado ao nível do mar com velocidade inicial $V_{0}=1 \mathrm{~km} / \mathrm{s}$ e $\Phi=45^{\circ}$.

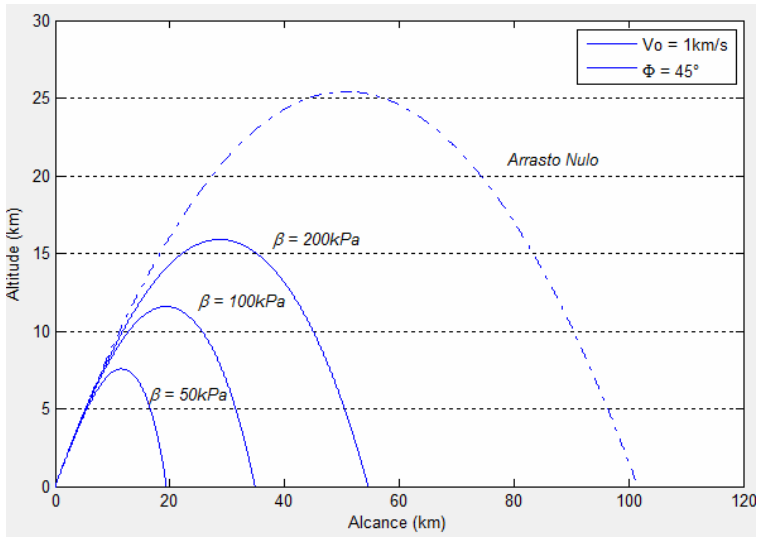

Figura 8. Efeito do arrasto para lançamento ao nível do mar.

A influência do coeficiente balístico no alcance ao decorrer do tempo de voo encontra-se na figura 9. Quanto menor o coeficiente balístico menor será a capacidade de alcance.

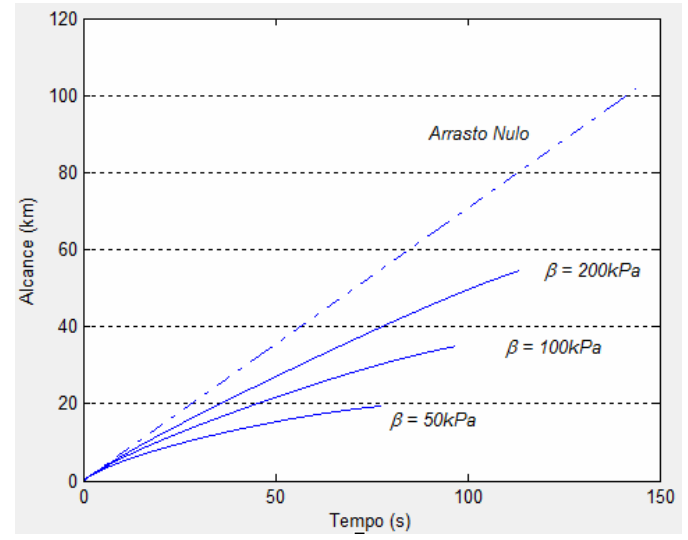

Figura 9. Alcance vs tempo para lançamento ao nível do mar.

A variação do valor absoluto da velocidade do míssil no decorrer do tempo encontra-se na figura 10 . Novamente, quanto menor for o coeficiente balístico mais acentuado será a queda na velocidade.

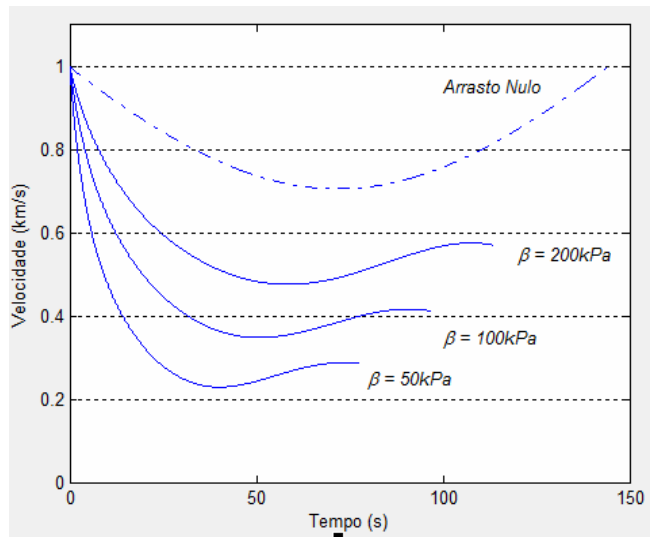

Figura 10. Velocidade vs tempo para lançamento ao nível do mar. 
Nota-se através da Figura 11 que o efeito do arrasto é reduzido consideravelmente para elevadas altitudes de lançamento. De maneira específica, os resultados das figuras 11,12 e 13 foram obtidos para as condições de $V_{0}=1 \mathrm{~km} / \mathrm{s}, \Phi=45^{\circ}$ e lançamento a $5 \mathrm{~km}$ de altitude.

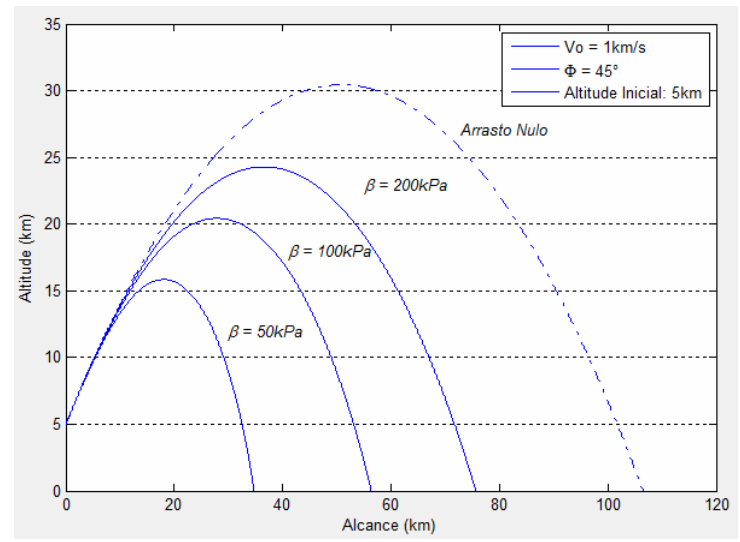

Figura 11. Redução do efeito do arrasto para lançamento a $5 \mathrm{~km}$ de altitude.

Na Figura 12, bem como para a Figura 9, nota-se que a relação entre o alcance e o tempo de voo deixa de ser linear conforme diminuímos o coeficiente balístico.

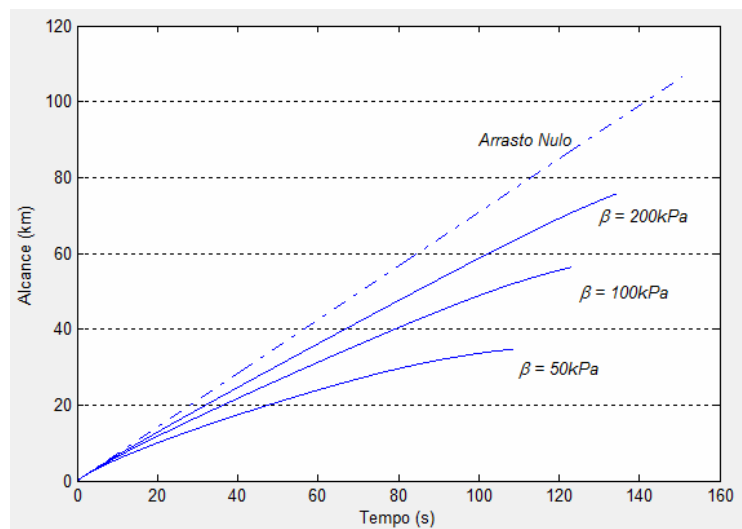

Figura 12. Alcance vs tempo para lançamento a $5 \mathrm{~km}$ de altitude.

A variação do valor absoluto da velocidade do míssil no decorrer do tempo encontra-se na Figura 13.

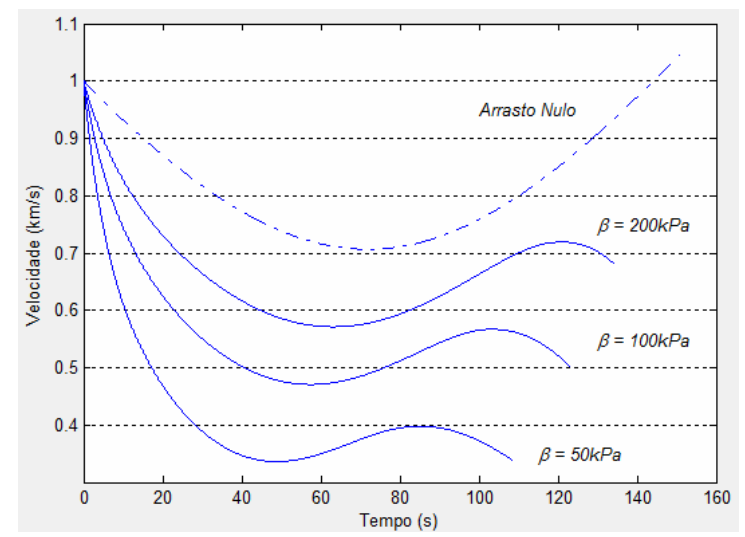

Figura 13. Velocidade vs tempo para lançamento a $5 \mathrm{~km}$ de altitude.

\section{Conclusão}

Através das simulações realizadas no presente trabalho fica evidente o efeito do arrasto atmosférico na trajetória balística do míssil. Deste modo quando maior for o $\beta$ menor será a influencia do arrasto. Além disso, o aumento na altitude da posição de lançamento tende a diminuir também os efeitos do arrasto na trajetória do míssil.

\section{Agradecimentos}

A Deus sobre todas as coisas. À minha família pelo apoio e motivação. Ao $\mathrm{CNPq}$ pela bolsa de pesquisa. A todos que de alguma maneira sempre me apoiaram.

\section{Referências Bibliográficas}

Bate, R.R; Muller, D.D; White, J. E.; "Fundamentals of Astrodynamics", Dover Publications, Nova York, 1971

Zarchan, P.; "Tactical and Strategic Missile Guidance", American Institute of Aeronautics and Astronautics, Virginia, 1997 